
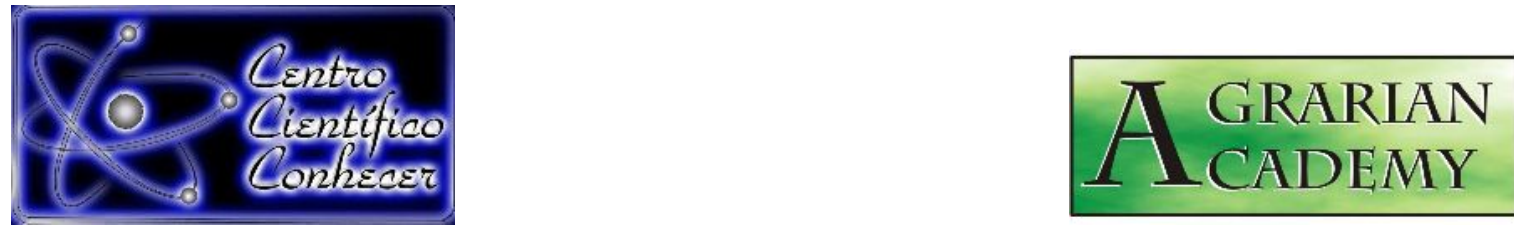

\title{
ANÁLISE DA EFICIÊNCIA POLÍNICA DE BISNAGUEIRA MEDIANTE DIFERENCIADOS MÉTODOS DE PIGMENTAÇÃO
}

Cyntia Beatriz Magalhães Farias ${ }^{1 *}$; Leila Pereira Neves Ramos ${ }^{2}$; Vanessa dos Santos de Mello ${ }^{3}$ Z Zélia Marques da Silva Radons Prestes ${ }^{4}$; Isane Vera Karsburg ${ }^{5}$

${ }^{1}$ Bióloga, formada pela da Universidade do Estado de Mato Grosso, Universidade do

Estado de Mato Grosso, Campus de Alta Floresta, MT - Brasil

${ }^{2}$ Bióloga, Mestranda pelo Programa de Pós Graduação em Genética e Melhoramento de Plantas, Universidade do Estado de Mato Grosso, Campus de Alta Floresta, MT - Brasil

${ }^{3}$ Mestre, pelo Programa de Pós Graduação em Genética e Melhoramento de Plantas, Universidade do Estado de Mato Grosso, Campus de Alta Floresta, MT Brasil

${ }^{4}$ Graduanda em Licenciatura Plena e Bacharelado em Ciências Biológicas, Universidade do Estado de Mato Grosso, Campus de Alta Floresta, MT - Brasil

${ }^{5}$ Docente da Faculdade de Ciências Biológicas e Agrárias, Programa de Pós Graduação em Genética e Melhoramento de Plantas, Universidade do Estado de Mato Grosso. 78.580-000 Alta Floresta, MT - Brasil

*cyntia_bmf@hotmail.com

Recebido em: 19/11/2018 - Aprovado em: 14/12/2018 - Publicado em: 25/12/2018 DOI: 10.18677/Agrarian_Academy_2018B1

Spathodea campanulata, da família Bignoniaceae, conhecida como tulipa africana é uma árvore originária da África, utilizada na medicina tradicional. No país é considerada planta ornamental. $O$ atual estudo verificou a fertilidade do grânulo de pólen de três acesso de $S$. campanulata, por meio do colorante 2,3,5 trifeniltetrazolio (TTC) em duas concentrações $(0,075 \%$ e $0,30 \%)$, exposto por $6,12,18$ e 24 horas, Reativo de Alexander, Lugol $1 \%$ e Verde Malaquita $1 \%$ no município de Alta Floresta-MT. Com a finalidade de confeccionar as lâminas utilizou-se uma pequena gota do pigmento em questão realizando-se a maceração das anteras, cobrindo com lamínula. A verificação das lâminas foi feita pela técnica de escaneamento em microscópio óptico em objetiva de 40x, 300 polens por lâmina em 10 repetições. Os resultados mostraram-se positivos verificando que para a viabilidade dos grânulos das populações em estudo de $S$. campanulata, os testes de pigmentação revelaramse eficientes ao diferenciar os polens ativos dos inativos. Mediante as médias do TTC, 12 horas foi o melhor horário para quantificar a exequibilidade dos polens da espécie para ambas concentrações $(0,075 \%$ e $0,30 \%)$. R. de Alexander, melhor estimou a atividade respiratória do pólen da espécie com médias superior a $76 \%$.

PALAVRAS-CHAVE: Corante, Polens, Tulipa Africana. 


\title{
ANALYSIS OF POLYMER EFFICIENCY OF Spathodea campanulata BY DIFFERENTIATED PIGMENTATION METHODS
}

\begin{abstract}
Spathodea campanulata, of the family Bignoniaceae, known as African tulipa is a tree native to Africa, used in traditional medicine. In Brazil it is considered ornamental plant. The present study verified the viability of three populations of $S$. campanulata by means of the 2,3,5-triphenyltetrazolium (TTC) dyes in two concentrations $(0.075 \%$ and $0.30 \%)$ exposed by $(6,12,18)$ and 24 hours), Alexander Reactive, Lugol 1\% and Malachite Green $1 \%$ in the city of Alta Floresta-MT. For the purpose of making the sheets, the dye in question was used to gout the maceration of the anthers, covering with a coverslip. The verification of the slides was done by optical scanning microscopy in objective of 40x, 300 pollen per slide in 10 repetitions. The results were positive for pollen viability of the populations studied in $S$. campanulata, and the pigmentation tests were efficient in distinguishing active pollen from inactive pollen. By means of TTC averages, 12 hours was the best time to quantify the viability of the species' pollens for both concentrations $(0.075 \%$ and $0.30 \%)$. Reactive of Alexander, better estimated the respiratory activity of pollen of the species with means superior to $76 \%$.
\end{abstract}

KEYWORDS: Dye, Pollens, African Tulip.

\section{INTRODUÇÃO}

A Spahodea campanulata (Bignoniaceae), é conhecida como Tulipa Africana, Chama-da-floresta ou Bisnagueira, uma árvore perene de médio e grande porte, originária da África, apresenta flores em formato de tulipa, com coloração vermelhasalaranjadas, seus botões florais possuem uma grande quantidade de líquido mucilaginoso, é polinizada por pássaros e morcegos, sendo também visitada por abelhas (KARTHIKA et al., 2013).

A S. campanulata foi introduzida no Brasil como uma planta ornamental (MODRO et al., 2011). É encontrada em várias partes da América do Sul e também na Índia, tem sido utilizada na medicina tradicional Africana. É cultivada para fins ornamental e útil na verificação de erosões do solo. A madeira é utilizada na indústria de papel. Esta espécie apresenta compostos químicos e potencial de uso como medicamentos antimicrobianos, cicatrização de feridas, anti-úlcera, antidiabéticose, anti-oxidante etc (BRINDHA et al., 2012).

Com a finalidade de dar suporte aos programas de genética e melhoramento de plantas, o conhecimento sobre as características florais, é de estrema importância para selecionar futuros progenitores (MARO et al., 2010). Para utilizar espécies de plantas em projetos de melhoramento genético depende da obtenção de cultivares superiores (TECHIO et al., 2006). Diante de tal importância é relevante que em um programa de melhoramento genético vegetal a técnica da eficiência polínica seja utilizada a fim de adquirir maior conhecimento sobre o material, assim podendo fazer seleções (BRAMBATTI et al., 2016).

Há causas que podem levar à infertilidade polínica, incluindo a idade do grão de pólen e também os fatores físicos, como a temperatura e umidade. A eficácia polínica demonstra a capacidade masculina de produção das espécies. Diante disso o objetivo deste trabalho foi fazer testes com variadas técnicas colorimétricas, pois ao serem empregadas, poderão estimar a eficácia do potencial do corante e viabilidade do pólen da espécie (KUHN, 2015). Diante da importância deste tipo de estudo o objetivo deste trabalho foi verificar a eficiência polínica de três acessos de S. campanulata. 


\section{METERIAL E MÉTODOS}

O corrente estudo foi realizado no Laboratório de Citogenética e Cultura de Tecidos Vegetais na UNEMAT, Campus de Alta Floresta. No experimento utilizou-se de botões florais de $S$. campanulata, coletados de três locais (Quadro 1).

QUADRO 1. Identificação dos locais de coleta dos botões em pré-antese das populações de Spathodea campanulata e dados de GPS.

\begin{tabular}{ccc}
\hline População & Local de Coleta & Coordenadas \\
\hline Pop.1 & Alta Floresta - MT & Sul: 0951'10,0152 "Oeste: $56^{\circ} 3^{\prime} 56,1924 "$ \\
Pop.2 & Alta Floresta - MT & Sul: 09051'8.3988 "Oeste: $56^{\circ} 3^{\prime} 49.23^{\prime \prime}$ \\
Pop.3 & Alta Floresta - MT & Sul: 0951'46.8108 "Oeste: $56^{\circ} 5^{\prime} 7.0584 "$ \\
\hline
\end{tabular}

Para o exame do corante 2,3,5 trifeniltetrazolio (TTC), nas concentrações $0,075 \%$ e $0,30 \%$ os lançamentos florais foram abertos com bisturi, no sentido de retirar as anteras, as quais foram fragmentadas com bastão de vidro para a soltura dos grânulos na solução, permanecendo expostos por quatro horários de exposição $\left(6,12,18\right.$ e 24 horas). $O$ conteúdo extraído ficou exposto à temperatura de $26^{\circ} \mathrm{C}$ no escuro. Na elaboração das lâminas adicionou-se o pigmento sobre a lâmina, logo o material foi coberto com uma lamínula. Para a investigação das lâminas utilizou-se o método de escaneamento visual de 300 partículas de pólen por lâmina, com 10 reproduções para cada tratamento. A viabilidade polínica foi marcada pela eficiência de pigmentação dos grãos de pólen, julgando-se viáveis os polens que apresentaram cor vermelha no protoplasma e inviáveis os que não coloriram.

Na preparação do experimento com os demais corantes: R. Alexander, Lugol $1 \%$ e V. Malaquita $1 \%$, As lâminas foram feitas pela técnica de esmagamento (GUERRA; SOUZA, 2002). A fertilidade dos polens foi fixada pela pigmentação dos grãos, foram considerados viáveis os polens que apresentaram o protoplasma com a coloração característica dos respectivos corantes e inviáveis os polens que não tiveram uma coloração definida.

Para as análises das lâminas foi adotado o método de varrição, sendo contados 300 grãos de polens por lâmina, com 10 repetências para cada tratamento, sendo as lâminas observadas em microscópio óptico binocular na objetiva de 40x. Os dados de viabilidade estimada com os corantes: 2,3,5 trifeniltetrazolio (TTC), R. de Alexander, Lugol 1\% e V. Malaquita 1\% foram submetidos a análise de variância e as médias comparadas pelo teste de tukey em nível de $5 \%$ de probabilidade. Os resultados das investigações foram apurados pelo programa $R$, versão 3.3.2 ( $R$ CORE TEAM, 2016), com o auxílio do pacote ExpDes, versão 1.1.2 (FERREIRA et al., 2013).

\section{RESULTADOS E DISCUSSÃO}

De acordo com a tabela 1, no estudo de atividade polínica com as populações Spathodea campanulata, observou-se que o produto concentrado de 2,3,5 de cloreto de Trifeniltetrazólio (TTC), contiveram diferença relevante para os horários 6,12,18 e $24 \mathrm{~h}$, podendo verificar que os testes de coloração mostraram-se eficientes ao diferenciar os polens viáveis dos inviáveis. Para as médias do TTC, 12 horas foi o melhor horário que estimou a viabilidade polínica para ambas as concentrações $(0,075 \%$ e $0,30 \%)$, com todas as médias superiores à $70 \%$.

Nos exames de Lauton et al. (2016) ao desenvolver um teste de fertilidade polínica com a linhagem de Jasmim manga, utilizando a solução 2,3,5 trifeniltetrazolio (TTC), nos horários de 6, 12, 18 e 24h, o horário de 12h, a 
concentração de $0,075 \%$ se apresentou com os melhores percentuais de grãos viáveis da espécie. Segundo Santos et al. (2014) em uma pesquisa de análise de viabilidade com a espécie Passiflora setacea, que se adotar menores concentrações $(0,075 \%)$, poderá proporcionar diminuição de gastos.

TABELA 1. Média da viabilidade polínica de três populações de Spathodea campanulata pela coloração em duas concentrações de TTC e quatro tempos de exposição.

\begin{tabular}{cccccc}
\hline \multirow{2}{*}{ Populações } & \multirow{2}{*}{ Concentrações } & \multicolumn{4}{c}{ Tempos } \\
\cline { 3 - 6 } & & $\mathbf{6}$ & $\mathbf{1 2}$ & $\mathbf{1 8}$ & $\mathbf{2 4}$ \\
\hline $\mathbf{0 1}$ & $0,075 \%$ & $82,43 \mathrm{a}$ & $83,39 \mathrm{a}$ & $80,66 \mathrm{a}$ & $82,16 \mathrm{a}$ \\
& $0,30 \%$ & $81,39 \mathrm{a}$ & $82,23 \mathrm{a}$ & $73,60 \mathrm{~b}$ & $82,29 \mathrm{a}$ \\
$\mathbf{0 2}$ & $0,075 \%$ & $82,93 \mathrm{a}$ & $77,16 \mathrm{~b}$ & $81,19 \mathrm{a}$ & $76,80 \mathrm{~b}$ \\
& $0,30 \%$ & $82,33 \mathrm{a}$ & $82,49 \mathrm{a}$ & $75,73 \mathrm{~b}$ & $81,16 \mathrm{a}$ \\
& $0,075 \%$ & $81,06 \mathrm{a}$ & $82,66 \mathrm{a}$ & $70,46 \mathrm{c}$ & $81,73 \mathrm{a}$ \\
$\mathbf{0 3}$ & $0,30 \%$ & $81,38 \mathrm{a}$ & $83,16 \mathrm{a}$ & $57,00 \mathrm{~d}$ & $82,76 \mathrm{a}$
\end{tabular}

CV (\%)

7,14

Médias seguidas de letras iguais nas linhas não diferem pelo teste Tukey em nível de 5\% de significância.

$\mathrm{Na}$ análise da espécie $S$. campanulata, ocorreu dissemelhança estatística entre as médias comparadas entre os corantes, podendo constatar que dentre os corantes utilizados: Reativo de Alexander, Lugol 1\% e Verde Malaquita 1\% aprestamaram resultados satisfatório. Ambos corantes apresentando médias altas, visto que, o R. de Alexander forneceu dados com maior listagem de efitividade dos polens em comparação ao Lugol 1\% e Verde malaquita 1\% (Tabela 2).

Lima et al. (2015) em análise da espécie Malpighia emarginata utilizaram os corantes Lugol e R. de Alexander, sendo que o Lugol expôs melhor precisão nos valores de exequibilidade polínica para a espécie. Silva et al., (2015), verificaram que a viabilidade de S. mombin com reativo de Alexander, lugol $1 \%$ e outros, foram superiores à $97 \%$, recomendando o uso para trabalhos com espécies nativas.

TABELA 2. Médias percentuais da viabilidade polínica das populações de Spahodea campanulata.

\begin{tabular}{ccccc}
\hline Corantes & \multicolumn{4}{c}{ Populações } \\
\cline { 2 - 5 } & $\mathbf{0 1}$ & $\mathbf{0 2}$ & $\mathbf{0 3}$ & CV (\%) \\
\hline Reativo de Alexander & $76,93 \mathrm{a}$ & $81,16 \mathrm{a}$ & $79,90 \mathrm{a}$ & 7,27 \\
Lugol 1\% & $82,36 \mathrm{a}$ & $78,59 \mathrm{a}$ & $34,60 \mathrm{~b}$ & 18,95 \\
Verde Malaquita 1\% & $81,56 \mathrm{a}$ & $79,16 \mathrm{ab}$ & $77,90 \mathrm{~b}$ & 3,93 \\
\hline
\end{tabular}

Médias seguidas de letras iguais nas linhas não diferem pelo teste Tukey em nível de $5 \%$ de significância.

Para o uso de diferentes métodos de pigmentação o 2,3,5 trifeniltetrazolio (TTC), R., Lugol $1 \%$ e V. Malaquita $1 \%$, houve resultados significativos entre a coloração dos polens viáveis e inviáveis (Figura1). O uso dos tonalizantes TTC $(0,30$ e $0,075 \%$ ) mostrou-se favoráveis quanto à distinção da cor dos grãos de pólen viáveis, a viabilidade polínica foi determinada pela capacidade de colorir os grãos, percebendo viáveis os polens que apresentaram cor do protoplasma de vermelho e inviáveis os que não tiveram coloração definida.

Os corantes: Reativo de Alexander, Lugol 1\% e Verde malaquita 1\%, também mostraram-se efetivos na distinção dos polens viáveis, quando os mesmos AGRARIAN ACADEMY, Centro Científico Conhecer - Goiânia, v.5, n.10; p. 42018 
apresentaram o protoplasma com o pigmento característico dos respectivos corantes e inviável os que não mostraram a cor definida, sendo o Reativo de Alexander, que melhor estimou a exequibilidade da espécie com médias acima de $76 \%$.

Os autores Ramos et al. (2017) ao testarem distintos métodos colorimétricos (2,3,5 trifeniltetrazolio (TTC) e o Lugol $2 \%$ ) obtiveram resultados significativos para ambos corantes, com três acessos de Myrciaria cauliflora, os testes de coloração mostraram-se ótimos para a espécie, os polens apresentaram nítidas diferenças na coloração, distinguindo viáveis de inviáveis.

Hister et al. (2016) ao desenvolverem um trabalho com Psidium cattleianum, puderam constatar que o reativo de Alexander mostrou-se mais eficaz na diferenciação entre os polens, na maioria dos acessos estudados com viabilidade polínica alta, sendo esta superior a $80,3 \%$.

Leite et al. (2018) verificaram que os corantes Lugol $2 \%$, Verde Malaquita e Reativo de Alexander, mostraram-se eficientes ao distinguir os polens viáveis dos inviáveis para a espécie S.paludosum e houveram altas taxas de viabilidade, no entanto eles não diferiram entre si.

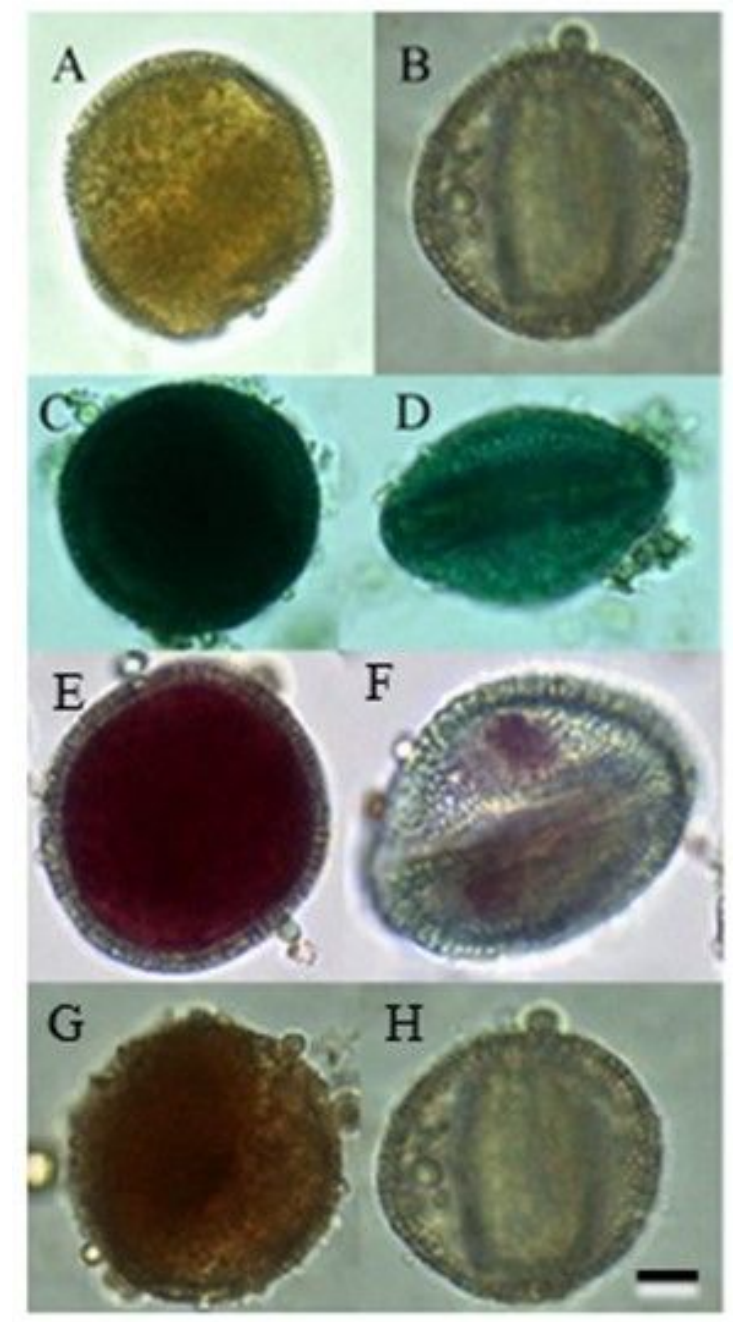

FIGURA 1. Grãos de pólen de Spathodea campanulata com uso de diferentes corantes: Lugol $1 \%$ a) viável b) inviável; Verde Malaquita $1 \%$ c) viável d) inviável; Reativo de Alexander e) viável f) inviável. Barra $=10 \mu \mathrm{m}$. 


\section{CONCLUSÕES}

$\mathrm{Na}$ averiguação da fertilidade dos gametas masculinos das populações de $S$. campanulata, os testes de coloração mostraram-se efetivos ao diferenciarem os polens.

Defronte as médias com 2,3,5 de cloreto de Trifeniltetrazólio (TTC), 12 horas foi o melhor horário para aferir a viabilidade polínica da espécie para ambas concentrações $(0,075 \%$ e $0,30 \%)$.

A viabilidade polínica de $S$. campanulata foi considerada alta para ambos corantes, Reativo de Alexander, Lugol 1\% e Verde malaquita 1\%, mas, o Reativo de Alexander, melhor estimou com médias aquém de $76 \%$.

\section{AGRADECIMENTOS}

Coordenação de Aperfeiçoamento de Pessoas de Nível Superior-Brasil (CAPES) - Cod de Financiamento 001.

\section{REFERÊNCIAS}

BRAMBATTI, A.; BRAMMER, S. P.; WIETHÖLTER, P.; JUNIOR, A. N. Estabilidade genética em triticale estimada pela viabilidade polínica. Arquivos do Instituto biológico. v.83, 1-7, e0802014, 2016. Disponível em:

<https://www.alice.cnptia.embrapa.br/bitstream/doc/1067829/1/ID440162016v83Arql nstBiole0802014.pdf>.

FERREIRA, E. B.; CAVALCANTI, P. P.; NOGUEIRA, D. A. ExpDes: Experimental Designs package. $R$ package version 1.1.2, 2013.

GUERRA, M.; SOUZA, M.J. Como observar cromossomos: um guia de técnicas em citogenética vegetal, animal e humana. Ribeirão Preto: FUNPEC, p. 201, 2002.

HISTER, C. A. L.; TEDESCO, S. B. Estimativa da viabilidade polínica de araçazeiro (Psidium cattleianum Sabine) através de distintos métodos de coloração. Revista brasileira de plantas medicinais, v. 18, n. 1, p. 135-141, 2016. Disponível em: <http://www.scielo.br/pdf/rbpm/v18n1/1516-0572-rbpm-18-1-0135.pdf>

Doi: 10.1590/1983-084X/15_081

KARTHIKA D, K.; MOHANRAJ, R. S.; DHANAKKODI, B. Mosquitocidial activities of Spathodea campanulata methanolic leaf extract against the dengue vector Aedes aegypti. Asian Journal of Plant Science and Research, v. 3, n. 4, p. 138-149, 2013. Disponível em:<www.pelagiaresearchlibrary.com>.

KUHN. A. W. Viabilidade polínica, Genotoxicidade, Efeito antiproliferativoe Composto fenólicos de PeltodonLongipes Kunth ExBenth. (Lamiaceae). Dissertação de Mestrado. Universidade Federal de Santa Maria-UFSM. Santa Maria/RS., $\quad 2015, \quad 58 \quad$ p. $\quad$ Disponível em: <https://repositorio.ufsm.br/bitstream/handle/1/4890/KUHN\%2c\%20ANDRIELLE\%20 WOUTERS.pdf?sequence $=1$ \&isAllowed $=\mathrm{y}>$. 
LAUTON, D. S.; SANTOS, A. C.; KARSBURG, I. V.; DAMASIO, J. F.; Viabilidade polínica de Jasmim Manga com corante tetrazólio. Ciência \& Tecnologia: Fatec-JB, Jabuticabal, v. 8, n.1, 2016. Disponível em:

$<w w w . c i t e c$.fatecjab.edu.br/index.php/files/article/download/1057/pdf $>$.

LEITE, D. M.; MACEDO, W. de A.; RAMOS, LEILA P. N.; MELLO, V. dos S. de; KARSBURG, I. V. Estudo da viabilidade polínica de Solanum paludosum Moric. (Solanaceae) por meio de distintos métodos colorimétricos. Agrarian academy, Centro Cientifico Conhecer - Goiania, v.5, n.9; p. 234 2018. DOI: 10.18677.

LIMA, J. dos S.; ROCHA, V. D. da.; TIAGO, A. V.; SANTOS, T. A. dos.; ROSSI, A. A. B. Influência do Horário de Coleta Sobre a Viabilidade de Grãos de Pólen em Acerola (Malpighia emarginata D C.). Enciclopédia Biosfera, Centro Científico Conhecer - Goiânia, v.11 n.21, 2015. Disponível em:

<http://www.conhecer.org.br/enciclop/2015b/multidisciplinar/influencia\%20do\%20hor ario.pdf>.

MARO, L. A. C.; CHAGAS, E. A.; PIO, R.; CHAGAS, P.; PASQUAL, M.; BETTIOL NETO, J. E.; COSTA, F. C. Composição do meio de cultura e condições ambientais para germinação de grãos de pólen de porta-enxertos de pereira. In: Embrapa Roraima-Artigo em anais de congresso (ALICE). In: CONGRESSO BRASILEIRO DE FRUTICULTURA, 21., 2010, Natal. Frutas: saúde, inovação e responsabilidade: anais. Natal: SBF, 2010. Disponível em: <http://submission.scielo.br/index.php/cr/article/viewFile/15650/2094>.

MODRO, A.; FRIDA, H.; MESSAGE, D.; LUZ, C. F. P. da.; NETO, J. A. A. M. Flora de Importância Polinífera Para Apis Mellifera (L.) Na Região De Viçosa, Mg. Revista Árvore, Viçosa-MG, v.35, n.5, p.1145-1153, 2011. Disponível em: < http://producao.usp.br/handle/BDPI/5728>.

BRINDHA, P.; NAGARAJAN, A.; SARALLA, R. P; R. NARENDRAN, R.; SRIDHARAN, K. A study on chemical and botanical standards of a traditional drug source Spathodea Campanulata beauv. Academiic Sciiences International. Journal of Pharmacy and Pharmaceutical Sciences ISSN- 0975-1491 Vol 4, Suppl 2, 2012.

em: <https://innovareacademics.in/journal/ijpps/Vol4Suppl2/43.pdf>.

R CORE TEAM. R: A language and environment for statistical computing. $\mathbf{R}$ Foundation for Statistical Computing, Vienna, Austria. URL https://www.R project.org/. 2016.

RAMOS, L. P. N.; ANDRÉ, V. L. S.; MELLO, V. dos. S. de.; DAMASIO, J. F.; KARSBURG, I. V. Estudo da Viabilidade Polínica de Myrciaria cauliflora (Mart.) O. Berg. Por Meio de Distintos Métodos de Coloração. Agrarian Academy, Centro Científico Conhecer - Goiânia, v.4, n.8, 2017. DOI: 10.18677.

SANTOS, M. L.; PORTO, A. C. M.; OLIVEIRA, A. C. Avaliação da viabilidade de embriões de plantas matrizeiras de maracujazeiro 'do sono' (Passiflora setacea DC) contrastantes quanto à capacidade de germinação. XX ENGENE - Encontro de 
Genética do Nordeste. Campina Grande - Paraíba - Brasil, 2014. Disponível em: <file://C:/Users/Administrador/Downloads/2548-7331-1-PB.pdf>.

SILVA, B. M. da; MORENO, E. C.; LIMA, J. dos S.; ROSSI, A. APa‥ B.; SOUZA, S. A. $M$. Potencial de testes colorimétricos na estimativa da viabilidade polínica em populações nativas de cajazeira. III Seminário de Biodiversidade e Agroecossistemas Amazônicos. Conservação de solos na Amazônia Meridional de13 a 16 de outubro de 2015 em Alta Floresta-MT- Universidade do Estado de Mato Grosso. Cáceres, v. 2, n. 1, 2015. ISSN 2358-5978. Disponível em:< http://tede2.uefs.br:8080/handle/tede/706>.

TECHIO, V. H.; CHAMMA DAVIDE, L.; PEDROZO, C. Â.; PEREIRA, V. A. Viabilidade do grão de pólen de acessos de capim-elefante, milheto e híbridos interespecíficos (capim-elefante x milheto). Acta Scientiarum. Biological Sciences, v. 28, n. $1,2006.2$ Disponível em: <http://www.redalyc.org/html/1871/187115870002/>. 